\title{
ON THE PATHWISE EXPONENTIAL STABILITY OF NON-LINEAR STOCHASTIC PARTIAL DIFFERENTIAL EQUATIONS
}

\author{
Tomás Caraballo and José Real \\ Dpto. Ecuaciones Diferenciales y Análisis Numérico. \\ Universidad de Sevilla. Apartado de Correos 1160. \\ 41080-SEVILLA (Spain)
}

December 15, 2009

\begin{abstract}
Sufficient conditions to get exponential stability for the sample paths (with probability one) of a non-linear monotone stochastic Partial Differential Equation are proved. In fact, we improve a stability criterion established in Chow [3] since, under the same hypotheses, we get pathwise exponential stability instead of stability of sample paths.
\end{abstract}

\section{INTRODUCTION AND PRELIMINARIES}

The aim of this paper is to give sufficient conditions in order to get exponential stability for the sample paths of certain stochastic PDE of evolution type.

In fact, we consider the following equation

$$
\left\{\begin{array}{l}
d x_{t}=A\left(t, x_{t}\right) d t+B\left(t, x_{t}\right) d w_{t}, \quad t>0 \\
x_{0}=h
\end{array}\right.
$$

where $A(t, \cdot)$ and $B(t, \cdot)$ are families of (non-linear) operators in Hilbert spaces and $w_{t}$ is a Hilbert Wiener process.

This equation has been studied by several authors over the last years. For instance, Pardoux [7], Ichikawa [6] and Caraballo [1] (among others) have established results on the existence and uniqueness of solutions. However, we are now interested in the analysis of the exponential stability for the paths of the trivial solution of (1). We can mention here that Haussmann [5] obtained pathwise exponential stability with probability one (w.p.1) for linear $A$ and $B$ and, Caraballo [2] generalized these results to the delayed case. Also, Ichikawa [6] proved similar results for the mild solution of the semilinear case, i.e. for linear $A$ and Lipschitz continuous $B$. At the 
same time, Chow [3] obtained asymptotic stability for the sample paths of (1) when $A$ and $B$ do not depend on $t$ and a coercivity condition holds. Recently, Chow and Menaldi [4] have obtained some estimates in exit probability for the strong solution of the semilinear problem. Nevertheless, we still have not found in the literature the study of exponential stability of paths in a more general case, i.e. for non-linear monotone $A$ and Lipschitz continuous $B$. So, we are going to analyze it in this paper. One of our results improves Theorem 5.2 from Chow [3] since, under the same hypotheses, we get exponential stability for the paths instead of the asymptotic stability that he obtains. First, we give sufficient conditions for the exponential stability in mean square of the trivial solution of (1). Next, we obtain exponential stability of paths (w.p.1). Our method is based in appying Itô's formula for a suitable function and using a coercivity condition. Consequently, we will observe how the coercivity condition may be regarded as a exponential stability criterion.

Now we are going to state our problem in a suitable form:

Let $V$ be a Banach space and $H, K$ real, separable Hilbert spaces such that

$$
V \hookrightarrow H \equiv H^{\prime} \hookrightarrow V^{\prime},
$$

where the injections are continuous and dense.

We denote by $\|\cdot\|,|\cdot|$ and $\|\cdot\|_{*}$ the norms in $V, H$ and $V^{\prime}$ respectively; by $\langle\cdot, \cdot\rangle$ the duality product between $V^{\prime}, V$, and by $(\cdot, \cdot)$ the scalar product in $H$.

Let $w_{t}$ be a Wiener process defined on the complete probability space $(\Omega, \mathcal{F}, P)$ and taking values in the separable Hilbert space $K$, with incremental covariance operator $W$. Let $\left(\mathcal{F}_{t}\right)_{t>0}$ be the $\sigma$-algebra generated by $\left\{w_{s}, 0 \leq s \leq t\right\}$, then $w_{t}$ is a martingale relative to $\left(\mathcal{F}_{t}\right)_{t \geq 0}$ and we have the following representation of $w_{t}$ :

$$
w_{t}=\sum_{i=1}^{\infty} \beta_{t}^{i} e_{i}
$$

where $\left(e_{i}\right)$ is an orthonormal set of eigenvectors of $W, \beta_{t}^{i}$ are mutually independent real Wiener processes with incremental covariance $\lambda_{i}>0, W e_{i}=\lambda_{i} e_{i}$ and $\operatorname{tr} W=$ $\sum_{i=1}^{\infty} \lambda_{i}$ (tr denotes the trace of an operator, see Pardoux [7]).

As an abuse of notation, we also use $|\cdot|$ for the norm in the linear continuous operator space $\mathcal{L}(K, H)$.

We denote by $I^{p}(0, T ; V)$, for $p>1$ and $T>0$, the space of $V$-valued processes $\left(x_{t}\right)_{t \in[0, T]}$ (we will write $x_{t}$ for short) measurable (from $[0, T] \times \Omega$ in $V)$, and satisfying:

1. $x(t)$ is $\mathcal{F}_{t}$-measurable a.e. in $t$ (in the sequel, we will write a.e.t.)

2. $E \int_{0}^{T}\left|x_{t}\right|^{p} d t<+\infty$.

It is not difficult to check that the space $I^{p}(0, T ; V)$ is a closed subspace of $L^{p}(\Omega \times[0, T], \mathcal{F} \otimes \mathcal{B}([0, T]), d P \otimes d t ; V)$, where by $\mathcal{B}([0, T])$ we denote the Borel $\sigma$-algebra. 
For short, we shall write $L^{2}(\Omega ; C(0, T ; H))$ instead of $L^{2}(\Omega, \mathcal{F}, d P ; C(0, T ; H))$, where $C(0, T ; H)$ denotes the space of continuous functions from $[0, T]$ to $H$.

Let $A(t, \cdot): V \rightarrow V^{\prime}$ be a family of non linear operators defined a.e.t. satisfying $A(t, 0)=0$ for all $t \geq 0$, and let $p>1$. (Note that we assume $A(t, 0)=0$ because we are going to restrict ourselves to the stability analysis for the trivial solution of (1)). We also consider a family of operators $B(t, \cdot): V \rightarrow \mathcal{L}(K, H)$ defined a.e.t., and satisfying:

(b.1) $B(t, 0)=0, \forall t \geq 0$

(b.2) Lipschitz condition: There exists $k_{1}$ such that

$$
|B(t, x)-B(t, y)| \leq k_{1}\|x-y\|, \quad \forall x, y \in V, \quad \text { a.e.t. }
$$

(b.3) Measurability:

$$
t \in(0, T) \rightarrow B(t, x) \in \mathcal{L}(K, H) \text { is Lebesgue-measurable } \forall x \in V, \forall T>0 .
$$

Since we are mainly interested in exponential stability questions, we will assume there exists a unique process

$$
x \in I^{p}(0, T ; V) \cap L^{2}(\Omega ; C(0, T ; H)), \quad \forall T>0,
$$

which is solution of (1). In other words, $x_{t}$ verifies the following integral equation in $V^{\prime}$ :

$$
x_{t}=x_{0}+\int_{0}^{t} A\left(s, x_{s}\right) d s+\int_{0}^{t} B\left(s, x_{s}\right) d w_{s}, t>0, P-\text { a.s. }
$$

where $x_{0}=h \in L^{2}\left(\Omega, \mathcal{F}_{0}, P ; H\right)$. Observe that, we can assure existence and uniqueness of solution for equation (2) if, for instance, the following conditions hold (see Pardoux [7]) 
(a.1) Coercivity: There exist $\alpha>0$ and $\lambda, \gamma \in$ such that:

$$
-2\langle A(t, x), x\rangle+\lambda|x|^{2}+\gamma \geq \alpha\|x\|^{p}+\left\|B(t, x) W^{1 / 2}\right\|_{2}, \quad \forall x \in V, \text { a.e.t. },
$$

where $\|\cdot\|_{2}$ denotes the Hilbert-Schmidt norm of nuclear operators, i.e.

$$
\left\|B(t, x) W^{1 / 2}\right\|_{2}=\operatorname{tr}\left(B(t, x) W B(t, x)^{*}\right)
$$

(a.2) Monotonicity:

$$
\begin{gathered}
-2\langle A(t, x)-A(t, y), x-y\rangle+\lambda|x-y|^{2} \geq\left\|(B(t, x)-B(t, y)) W^{1 / 2}\right\|_{2}, \text { forallx, } y \in \\
V, \text { a.e.t. }
\end{gathered}
$$

(a.3) Boundedness: There exists $c>0$ :

$$
\|A(t, x)\|_{*} \leq c\|x\|^{p-1}, \quad \forall x \in V \text {, a.e.t. }
$$

(a.4) Hemicontinuity:

The map $\theta \in \rightarrow\langle A(t, x+\theta y), z\rangle \in$ is continuous $\forall x, y, z \in V$, a.e.t.

(a.5) Measurability:

$t \in(0, T) \rightarrow A(t, x) \in V^{\prime}$ is Lebesgue - measurable $\forall x \in V$, a.e.t., $\forall T>0$.

\section{THE MAIN RESULTS}

We note that there exists a positive constant $\beta$ such that

$$
|x| \leq \beta\|x\| \quad \forall x \in V .
$$

Now, we state the exponential stability for the second moment of $x_{t}$, solution of (2).

TheOrem 2.1 Assume conditions (b.1)-(b.3) and (a.1). Then, there exists $r>0$ such that

$$
E\left|x_{t}\right|^{2} \leq E\left|x_{0}\right|^{2} e^{-r t} \quad \forall t \geq 0,
$$

if either one of the following hypotheses holds:
(a) $\lambda<0, \gamma \leq 0 \quad(\forall p>1)$
(b) $\lambda \beta^{2}-\alpha<0, \gamma \leq 0 \quad(p=2)$. 
Proof. We apply Itô's formula (see Pardoux [7], Caraballo [1]) for the function $\left.e^{\alpha t} \cdot\right|^{2}$ and the process $x_{t}$, where $r>0$ is such that $r+\lambda<0$ in case $(a)$ or $(r+\lambda) \beta^{2}-\alpha<0$ in case (b) (we note that there exists such $r$ since the maps $r \rightarrow r+\lambda, r \rightarrow(r+\lambda) \beta^{2}-\alpha$ are continuous and (a) or (b) holds).

We then obtain

$$
\begin{aligned}
e^{r t}\left|x_{t}\right|^{2}-\left|x_{0}\right|^{2}= & r \int_{0}^{t} e^{r s}\left|x_{s}\right|^{2} d s+2 \int_{0}^{t} e^{r s}\left\langle x_{s}, A\left(s, x_{s}\right)\right\rangle d s \\
& +2 \int_{0}^{t} e^{r s}\left\langle x_{s}, B\left(s, x_{s}\right) d w_{s}\right\rangle \\
& +\int_{0}^{t} e^{r s} \operatorname{tr}\left(B\left(s, x_{s}\right) W B\left(s, x_{s}\right)^{*}\right) d s .
\end{aligned}
$$

Now, since $\int_{0}^{\cdot} e^{r s}\left\langle x_{s}, B\left(s, x_{s}\right) d w_{s}\right\rangle$ is a continuous local martingale (see Caraballo [1] and Chow [3]), it follows

$$
E\left(\int_{0}^{t} e^{r s}\left\langle x_{s}, B\left(s, x_{s}\right) d w_{s}\right\rangle\right)=0 .
$$

Therefore, from (a.1) and (4) we can deduce

$$
\begin{aligned}
e^{r t} E\left|x_{t}\right|^{2} \leq & E\left|x_{0}\right|^{2}+(r+\lambda) \int_{0}^{t} e^{r s} E\left|x_{s}\right|^{2} d s \\
& -\alpha \int_{0}^{t} e^{r s} E\left\|x_{s}\right\|^{p} d s+\gamma\left(\frac{e^{r t}-1}{r}\right) .
\end{aligned}
$$

Now, if (a) holds, (5) yields

$$
e^{r t} E\left|x_{t}\right|^{2} \leq E\left|x_{0}\right|^{2},
$$

and if we suppose (b), (5) implies

$$
\begin{aligned}
e^{r t} E\left|x_{t}\right|^{2} & \leq E\left|x_{0}\right|^{2}+\left[(r+\lambda) \beta^{2}-\alpha\right] \int_{0}^{t} e^{r s} E\left\|x_{s}\right\|^{2} d s \\
& \leq E\left|x_{0}\right|^{2} .
\end{aligned}
$$

So, the proof is complete.

Now, we are going to establish that the exponential stability of the second moment implies the exponential stability of the sample paths w.p.1. First, we need the following result. 
Lemma 2.1 Assume the solution $x_{t}$ of problem (2) satisfies (3). Then, there exist positive constants $c_{1}, c_{2}$ such that

$$
\begin{aligned}
& \text { (a) } \int_{\tau}^{t} E\left|B\left(s, x_{s}\right)\right|^{2} d s \leq c_{1} E\left|x_{0}\right|^{2} e^{-r \tau}, 0 \leq \tau \leq t \\
& \text { (b) } E\left(\sup _{0 \leq t<+\infty}\left|x_{t}\right|^{2}\right) \leq c_{2} E\left|x_{0}\right|^{2} .
\end{aligned}
$$

Proof. First, applying Itô's formula as in Theorem 2.1 we get

$$
e^{r t} E\left|x_{t}\right|^{2} \leq E\left|x_{0}\right|^{2}+\left[(\lambda+r) \beta^{2}-\alpha\right] \int_{0}^{t} e^{r s} E\left\|x_{s}\right\|^{2} d s, \forall t \geq 0 .
$$

Since $(\lambda+r) \beta^{2}-\alpha<0$, it follows

$$
\int_{0}^{t} e^{r s} E\left\|x_{s}\right\|^{2} d s \leq \frac{1}{\alpha-(\lambda+r) \beta^{2}} E\left|x_{0}\right|^{2}, \quad \forall t \geq 0 .
$$

Now, (6) and (b.2) yield

$$
\int_{0}^{t} e^{r s} E\left|B\left(s, x_{s}\right)\right|^{2} d s \leq \frac{k_{1}}{\alpha-(\lambda+r) \beta^{2}} E\left|x_{0}\right|^{2} \equiv c_{1} E\left|x_{0}\right|^{2}, \quad \forall t \geq 0
$$

and, for $0 \leq \tau \leq t$, we get

$$
\int_{\tau}^{t} e^{r s} E\left|B\left(s, x_{s}\right)\right|^{2} d s \leq c_{1} E\left|x_{0}\right|^{2}
$$

and

$$
e^{-r \tau} \int_{\tau}^{t} e^{r s} E\left|B\left(s, x_{s}\right)\right|^{2} d s \leq c_{1} E\left|x_{0}\right|^{2} e^{-r \tau}, \quad 0 \leq \tau \leq t
$$

Therefore

$$
\int_{\tau}^{t} E\left|B\left(s, x_{s}\right)\right|^{2} d s \leq \int_{\tau}^{t} e^{r(s-\tau)} E\left|B\left(s, x_{s}\right)\right|^{2} d s \leq c_{1} E\left|x_{0}\right|^{2} e^{-r \tau}, \quad 0 \leq \tau \leq t,
$$

and hence (a) is proved. Next, Itô's formula for $\left|x_{t}\right|^{2}$ and the coercivity condition imply

$$
\begin{aligned}
\left|x_{t}\right|^{2}= & \left|x_{0}\right|^{2}+\int_{0}^{t}\left\langle x_{s}, A\left(s, x_{s}\right)\right\rangle d s+2 \int_{0}^{t}\left\langle x_{s}, B\left(s, x_{s}\right) d w_{s}\right\rangle \\
& +\int_{0}^{t} \operatorname{tr}\left(B\left(s, x_{s}\right) W B\left(s, x_{s}\right)^{*}\right) d s \\
\leq & \left|x_{0}\right|^{2}+|\lambda| \int_{0}^{t}\left|x_{s}\right|^{2} d s+2\left|\int_{0}^{t}\left(x_{s}, B\left(s, x_{s}\right), d w_{s}\right)\right|,
\end{aligned}
$$


and so,

$$
\begin{aligned}
E\left[\sup _{t \in[0, T]}\left|x_{t}\right|^{2}\right] \leq & E\left|x_{0}\right|^{2}+|\lambda| \int_{0}^{T} E\left|x_{t}\right|^{2} d t \\
& +2 E\left[\sup _{t \in[0, T]}\left|\int_{0}^{t}\left(x_{s}, B\left(s, x_{s}\right) d w_{s}\right)\right|\right] .
\end{aligned}
$$

Now, we estimate the terms on the right-hand side of (7).

$$
|\lambda| \int_{0}^{T} E\left|x_{s}\right|^{2} d s \leq|\lambda| \int_{0}^{T} e^{-r s} E\left|x_{0}\right|^{2} d s \leq \frac{|\lambda|}{r} E\left|x_{0}\right|^{2}, \quad \forall T>0 .
$$

Using Burkholder-Davis-Gundy's inequality we obtain:

$$
\begin{aligned}
E\left[\sup _{t \in[0, T]}\left|\int_{0}^{t}\left(x_{s}, B\left(s, x_{s}\right) d w_{s}\right)\right|\right] & \leq k_{3} E\left[\left(\operatorname{tr}(W) \int_{0}^{T}\left|x_{s}\right|^{2}\left|B\left(s, x_{s}\right)\right|^{2} d s\right)^{1 / 2}\right] \\
& \leq k_{4} E\left[\left(\int_{0}^{T}\left|x_{s}\right|^{2}\left|B\left(s, x_{s}\right)\right|^{2} d s\right)^{1 / 2}\right] \\
& \leq k_{5} E\left[\sup _{s \in[0, T]}\left|x_{s}\right|\left[\int_{0}^{T}\left|B\left(s, x_{s}\right)\right|^{2} d s\right]^{1 / 2}\right] \\
& \leq \frac{1}{2} E\left[\sup _{t \in[0, T]}\left|x_{t}\right|^{2}\right]+k_{6} \int_{0}^{T} E\left|B\left(s, x_{s}\right)\right|^{2} d s \\
& \leq \frac{1}{2} E\left[\sup _{t \in[0, T]}\left|x_{t}\right|^{2}\right]+k_{7} E\left|x_{0}\right|^{2}
\end{aligned}
$$

since (a) holds.

Finally, as $k_{7}$ does not depend on $T$, we can obtain (b) from (7) - (9) and Lebesgue's theorem.

Theorem 2.2 Assume the hypotheses in Theorem 2.1. Then, there exist positive constants $\xi, \eta$ and a subset $\Lambda \subset \Omega$ with $P(\Lambda)=0$ such that, for each $\omega \notin \Lambda$, there exists a positive number $T(\omega)$ such that the following holds:

$$
\left|x_{t}(\omega)\right|^{2} \leq \eta E\left|x_{0}\right|^{2} e^{-\xi t} \quad \forall t \geq T(\omega) .
$$

Proof. We only sketch the proof because it is similar to the linear case one (see Haussmann [5] and Caraballo [2], for details).

First, we apply Itô's formula. As $\gamma \leq 0$ and coercivity holds, we obtain:

$$
\left|x_{t}\right|^{2} \leq\left|x_{N}\right|^{2}+|\lambda| \int_{N}^{t}\left|x_{s}\right|^{2} d s+2\left|\int_{N}^{t}\left(x_{s}, B\left(s, x_{s}\right) d w_{s}\right)\right|
$$


for $t \geq N$, where $N$ is a natural number.

Next, if $I_{N}$ denotes the interval $[N, N+1]$ we have:

$$
\begin{aligned}
\sup _{t \in I_{N}}\left|x_{t}\right|^{2} \leq & \left|x_{N}\right|^{2}+|\lambda| \int_{N}^{N+1}\left|x_{s}\right|^{2} d s \\
& +2 \sup _{t \in I_{N}}\left|\int_{N}^{t}\left(x_{s}, B\left(s, x_{s}\right) d w_{s}\right)\right|
\end{aligned}
$$

and so,

$$
\begin{aligned}
P\left[\sup _{t \in I_{N}}\left|x_{t}\right|^{2} \geq \varepsilon_{N}^{2}\right] \leq & P\left[\left|x_{N}\right|^{2} \geq \varepsilon_{N}^{2} / 3\right]+P\left[|\lambda| \int_{N}^{N+1}\left|x_{s}\right|^{2} d s \geq \varepsilon_{N}^{2} / 3\right] \\
& +P\left[2 \sup _{t \in I_{N}}\left|\int_{N}^{t}\left(x_{s}, B\left(s, x_{s}\right) d w_{s}\right)\right| \geq \varepsilon_{N}^{2} / 3\right]
\end{aligned}
$$

where $\varepsilon_{N}=E\left|x_{0}\right| e^{-r N / 4}$.

Now, we can estimate the terms on the right-hand side of (12) using Kolmogorov's inequality and (3) for the first two terms and we also use the inequalities of Burkholder-Davis-Gundy and Holder and Lemma 2.1 for the last. We then obtain

$$
P\left[\sup _{t \in I_{N}}\left|x_{t}\right|^{2} \geq \varepsilon_{N}^{2}\right] \leq k_{8} e^{-r N / 4},
$$

and finally, Borel-Cantelli's lemma completes the proof.

Remark. We can observe how the coercivity condition (with (a) or (b)) implies pathwise exponential stability for the solutions of problem (2). However, Chow [3] only obtains asymptotic stability under the same hypotheses. We also note that assumption (iii) in [3, Theorem 5.2] never holds because from (a.1) with $x=0$ it follows $\gamma \geq 0$. Consequently, in (a) and (b) we can set $\gamma=0$ instead of $\gamma \leq 0$ and the same in Chow's Theorem 5.2.

\section{EXAMPLES}

First, we note that, in the examples given in Chow [3], the trivial solution has exponentially stable paths instead of the asymptotic stability property stated there. Next, we are going to give a different example.

Let $\mathcal{O}$ be an open, bounded subset in ${ }^{N}$ with regular boundary and let $2<p<+\infty$. We consider the Sobolev space $V=W_{0}^{1, p}(\mathcal{O}), H=L^{2}(\mathcal{O})$ with their usual inner products, and the monotone operator $A: V \mapsto V^{\prime}$ defined as

$$
\langle A u, v\rangle=-\sum_{i=1}^{N} \int_{\mathcal{O}}\left|\frac{\partial u(x)}{\partial x_{i}}\right|^{p-2} \frac{\partial u(x)}{\partial x_{i}} \frac{\partial v(x)}{\partial x_{i}} d x-\int_{\mathcal{O}} a(x) u(x) v(x) d x \quad \forall u, v \in V,
$$


where $a \in L^{\infty}(\mathcal{O})$ satisfies $a(x) \geq \hat{a}>0$, a.e. $x \in \mathcal{O}$. We also consider $B(u)=$ $g(u), u \in V$ where $g: \mapsto$ is Lipschitz continuous with constant $L$ such that $L^{2}<$ $2 \hat{a}$ and $g(0)=0$. Finally, let $w_{t}$ be a standard real Wiener process (so, $K=$ and $W=1$ ). Then, although condition (a.3) does not hold when $\|u\| \leq 1$, we can assure there exists a unique solution of $(2)$, for each $u_{0} \in L^{p}\left(\Omega, \mathcal{F}_{0}, P ; H\right)$ (see Pardoux [7], Theorem 4.1, p. 126). In this case, (a.1) holds with $\gamma=0, \lambda=$ $-\varepsilon<0, p>2, \alpha=2$, where $\varepsilon>0$ is such that $L^{2} \leq 2 \hat{a}-\varepsilon$. Consequently, we get asymptotic exponential stability for the paths of the trivial solution, $u$, of a problem which can be interpreted as follows:

$$
\left\{\begin{aligned}
d u(t, x)= & \left(\sum_{i=1}^{N} \frac{\partial}{\partial x_{i}}\left(\left|\frac{\partial u(t, x)}{\partial x_{i}}\right|^{p-2} \frac{\partial u(t, x)}{\partial x_{i}}\right)-a(x) u(t, x)\right) d t \\
& +g(u(t, x)) d w_{t}, \text { a.e. in }(0,+\infty) \times \mathcal{O} \\
u(0, x)= & u_{0}(x), \text { a.e. in } \mathcal{O} \\
u(t, x)= & 0, \text { a.e. in }(0,+\infty) \times \partial \mathcal{O}
\end{aligned}\right.
$$

\section{References}

[1] T. Caraballo, Existence and Uniqueness of Solutions for Non-Linear Stochastic Partial Differential Equations, Collect. Math. $\underline{42}, 1$ (1991), 51-74.

[2] T. Caraballo, Asymptotic Exponential Stability of Stochastic Partial Differential Equations with Delay, Stochastics and Stochastics Reports, $\underline{33}$ (1990), $27-47$.

[3] P. L. Chow, Stability of Nonlinear Stochastic-Evolution Equation, J. Math. Anal. Appl., $\underline{89}$ (2) (1982), 400-419.

[4] P. L. Chow and J. L. Menaldi, Exponential Estimates in Exit Probability for some Diffusion Process in Hilbert Spaces, Stochastics and Stochastics Reports, $\underline{29}$ (1990), 377-393.

[5] U. G. Haussmann, Asymptotic Stability of the Linear Itô Equation in InfiniteDimension, J. Math. Anal. Appl., $\underline{65}$ (1978), 219-235.

[6] A. Ichikawa, Stability of Semilinear Stochastic Evolution Equations, J. Math. Anal. Appl., $\underline{90}$ (1982), 12-44.

[7] E. Pardoux, Equations aux Dérivées Partielles Stochastiques Nonlinéaires Monotones, Thesis, Université Paris Sud, 1975. 